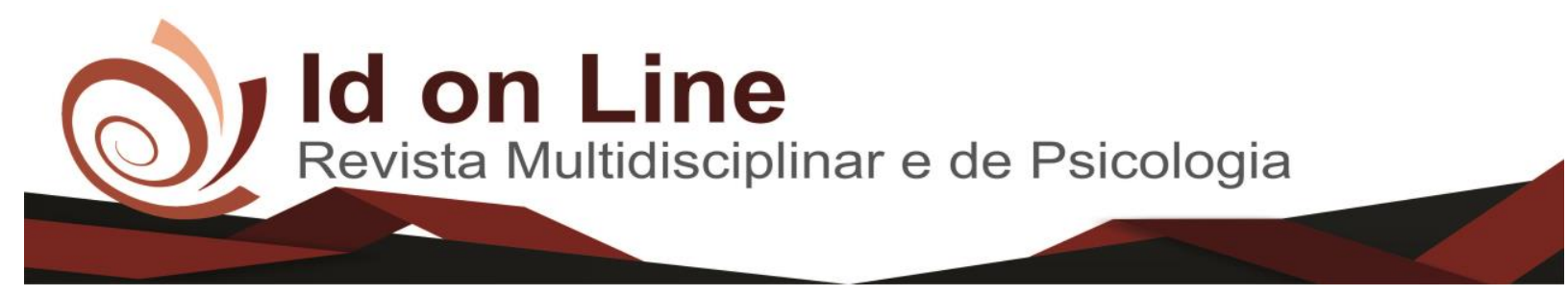

Artigo

\title{
Análise do efeito da Mobilização Neural na dor lombar em pacientes com hérnia de disco
}

\author{
Adirlane Moraes Ferreiral; ${ }^{\text {; Virgilio Santana Júnior }}{ }^{2}$
}

\begin{abstract}
Resumo: O objetivo deste estudo foi analisar a eficácia da Mobilização Neural no tratamento da dor lombar em pacientes com hérnia de disco lombar. Trata-se de um estudo analítico, realizado em 8 indivíduos, independente do sexo com aplicação do questionário sociodemográfico, Mcgill, Start Back e a Escala Visual Analógica para identificação das variáveis do estudo. O tratamento foi realizado com a mobilização neural em 16 sessões e 2 vezes por semana. Foram feitas análises descritivas das variáveis contínuas, como frequências absolutas e relativas, média, desvio-padrão, mínimo e máximo, para analise estatística utilizou-se o teste de normalidade de Kolmogorov-Smirnov. Para avaliar a comparação aplicaram-se o teste t de Student pareado e independente, e a análise de variância (ANOVA), com diferenciação das médias utilizando o teste Tukey. Para todos os testes estatísticos, utilizou-se o nível de significância de 5\%. A mobilização neural mostrou-se eficaz no tratamento da dor lombar em pacientes com hérnia de disco e na mobilidade funcional.
\end{abstract}

Palavras-chave: Hérnia Discal. Lombalgia. Mobilização Neural

\section{Analysis of the effect of Neural Mobilization on lower back pain in patients with disc herniation}

\begin{abstract}
This study aimed to analyze the effectiveness of Neural Mobilization in the treatment of lower back pain in patients with lumbar disc herniation. This is an analytical study, carried out in 8 individuals regardless of the gender with application of the sociodemographic questionnaire, Macgill, Start Back and Visual Analog Scale to identify the study variables. The treatment was executed with neural mobilization, for 16 sessions, twice a week. Descriptive analyzes were performed of continuous variables, such as absolute and relative frequencies, mean, standard deviation, minimum and maximum. The normality test performed was the Kolmogorov-Smirnov. The comparative analyzes were applied the Student's t test paired and independent, and analysis of variance (ANOVA), with differentiation of the means using the Tukey test. For all statistical tests, the significance level of 5\% was used. Neural mobilization in the treatment of lower back pain and functional mobility has been shown to be effective.
\end{abstract}

Keywords: Herniated Disc. Lower Back Pain. Neural Mobilization

\footnotetext{
1 Discente graduanda do curso de Fisioterapa pela Faculdade Independente do Nordeste- Fainor. http://www.fainor.com.br, Vitoria da Conquista-BA, Brasil. dilamoraesfisio@ hotmail.com

${ }^{2}$ Fisioterapeuta. Docente da FAINOR e FTC em Vitória da Conquista/BA. Especialista em terapia manual e postural - CESUMAR-PR.

Autor correspondente: Virgilio Santana Junior, Rua Hormindo Barros, 244; Bairro Candeias; CEP 45029-094; Vitoria da Conquista/BA; virgiliofisio@hotmail.com (77)98807-8862.
} 


\section{Introdução}

A Lombalgia é uma sintomatologia de incapacidade, que pode gerar um aumento dos níveis de absenteísmo no trabalho, uma vez que esses níveis são elevados podem causar maiores custos para o sistema de saúde, afetando 70 a $80 \%$ da população. Torna-se crônica em 10 a 15\% da população, esse impacto na população gera incapacidades nas atividades laborais, sociais e em família, podendo ser desencadeada por uma alteração do disco vertebral como, por exemplo, uma hérnia de disco ou alterações musculoesqueléticas. No Brasil, as manifestações da coluna estão se destacando como principais causas de pagamentos de auxílio-doença e a terceira causa de beneficio por invalidez, (BIANCHL et al.,2016).

A hérnia de disco pode ocorrer por vários micros traumas de uma forma indireta ou direta na coluna que vão, em certo período de tempo, destruindo as composições do disco intervertebral. Essas lesões podem causar sintomatologias ou podem ser assintomáticas o que depende do local comprometido, da extensão, da tipologia e do grau (MENDONÇA; ANDRADE, 2016).

A Hérnia de Disco Lombar (HDL) é uma patologia muito frequente no aparelho locomotor que atinge a região inferior da coluna que esta relacionada á ruptura do anel fibroso, consequentemente deslocamento da massa central do disco. Em sua maioria ocorrem entre L4L5 e L5-S1 sendo o local de apoio dos movimentos dos troncos e membros inferiores e em função da maior mobilidade da região lombossacra com auxilio da musculatura envolvida tanto profunda como superficial (SIQUEIRA et al., 2014).

A coluna lombar é basicamente estabilizada pelos músculos multifidos e o transverso do abdome eles são os principais estabilizadores. A fraqueza de controle dessa musculatura, esta entre as causas de dor na coluna lombar, nesses casos é um fator preditivo para alterações funcionais, tensionamentos e quadro álgico. Qualquer desequilíbrio lombar gera disfunções no recrutamento dos músculos que estabilizam a coluna lombar, dessa forma, em uma desordem musculoesquelética, os músculos próximos da articulação lesada são atingidos, podendo gerar uma redução da força e consequentemente atrofia muscular, aumentando o nível de instabilidade (KOBILL et al., 2017).

De acordo com a alta prevalência de lombalgias diversas técnicas de tratamento vêm sendo aplicada para minimizar os danos dessas disfunções entre elas esta a Mobilização Neural (FERREIRA; OLIVEIRA, 2016). 
A Mobilização Neural (MN) é uma terapia manual que restitui o movimento e a elasticidade do sistema nervoso, gerando um melhor funcionamento das regiões musculoesqueléticas sem suas respectivas funções. A reabilitação se da através de movimentos oscilantes ou sustentados, sempre na direção dos nervos periféricos que apresentam limitação no deslizamento, com isso os sinais de tensão neural apareceram durante os testes neurais específicos para cada região (FREITAS et al., 2015).

Essa pesquisa tem como principal interesse analisar a eficácia da técnica de Mobilização Neural em indivíduos com hérnia de disco na coluna lombar, para que contribua no direcionamento da reabilitação.

\section{Metodologia}

O presente estudo foi do tipo quantitativo, com delineamento transversal e caráter analítico, sendo do tipo não probabilístico por conveniências, realizado no departamento de Fisioterapia da Faculdade Independente do Nordeste (FAINOR) no período de agosto e setembro de 2017. O presente estudo atendeu às normas para a realização de pesquisa em seres humanos e cumpriu as diretrizes da Resolução 466/12 do Conselho Nacional de Saúde, sendo o estudo aprovado pelo Comitê de Ética da Faculdade Independente do Nordeste, n. 2132342.

A amostra foi constituída por oito indivíduos de ambos os sexos na faixa etária de 30 a 70 anos escolhida por análise não probabilística por conveniência. Foram incluídos os indivíduos que realizam atendimento fisioterapêutico no Núcleo de Fisioterapia da FAINOR que se dispôs a participar da pesquisa como voluntários e assinaram o termo de consentimento livre e esclarecido. Foram empregados como critérios de exclusão: pacientes de pós- cirúrgico e pacientes com artrodeses que pudessem interferir nos resultados do trabalho proposto ou qualquer fator de contraindicação para receber a técnica de Mobilização Neural.

Os dados foram coletados através de um questionário, composto por variáveis sociodemográficas como idade, sexo, peso, altura, escolaridade, localização da hérnia de disco e realizado a goniometria de flexão da coluna lombar. A goniometria é um método manual comumente utilizado por fisioterapeutas com objetivo de avaliar a amplitude de movimento, pois alem de ser de baixo custo é um instrumento de fácil manuseio, sendo assim um método de fácil acesso na prática clinica (KODAMA et al., 2017). Para avaliar dor usou a Escala Visual 
Analógica (EVA) um método validado que aprecia de forma quantitativa a presença e a intensidade de dor do indivíduo. Constituída por reta de $10 \mathrm{~cm}$ que mensura de zero a dez, graduando zero como ausência de dor e dez intensidade máxima de dor (STORCH et al., 2015).

Em seguida foi aplicado o questionário de Mcgill de Dor sendo do tipo multidimensional, através de uma avaliação de intensidade e escalas unidimensionais. Ela caracteriza a dor em três categorias: sensorial, afetiva e avaliativa subjetiva, além da intensidade e localização. A categoria sensorial descreve a qualidade da dor em termos temporais, espaciais, de pressão, temperatura, entre outras. A categoria afetiva avalia a qualidade da experiência da dor em termos de tensão, medo, temor, recuo e propriedades autonômicas. A avaliativa descreve uma avaliação geral da dor (MENDES et al., 2016).

Posteriormente foi aplicado o Questionário Start Back (SBST) um instrumento que classifica alto, médio e baixo risco de fatores psicossociais e a classificação de pacientes com dor lombar ou lombociatalgia, é constituído de nove itens, relacionado a dor referida, disfunção e comorbidades e cinco itens compõe a subescala psicossocial ( itens 5 a 9). Já o nono item apresenta cinco opções de resposta: "Nada, Pouco, Moderada, Muito, Extremamente". Se a pontuação total for entre 0-3 pontos, o paciente é classificado como de baixo risco. Para valores maiores que 3 na pontuação total, considera-se então a pontuação da subescala psicossocial. Se a pontuação dessa subescala for $\leq 3$ pontos, o paciente é classificado como de médio risco e, se for $>3$ pontos, encaixa-se no grupo de alto risco (PILZ et al., 2014).

No que tange a coleta de dados foi dividida em três passos: avaliação inicial (prémobilização), aplicação da mobilização neural e reavaliação (pós-mobilização neural). $\mathrm{O}$ paciente em ortostase o avaliador realizou através do goniômetro clínico a medição do angulo de flexão de tronco, tendo como pontos de referência: braço fixo do goniômetro colocado perpendicularmente ao solo no nível da crista ilíaca e o braço móvel do ao completar o movimento, ao longo da linha axilar média do tronco com objetivo de identificar a restrição na amplitude de movimento em graus, pré-mobilização e pós-mobilização neural do nervo isquiático.

Os participantes foram submetidos ao protocolo de $\mathrm{MN}$ para o nervo isquiático por 8 semanas, sendo atendidos 2 vezes na semana totalizando 16 sessões. O protocolo foi aplicado pelo avaliador com paciente posicionado em decúbito dorsal na maca, um membro inferior em flexão de mais ou menos $30^{\circ}$ e consistiu em movimentos oscilatórios por 2 minutos por quarto repetições em cada membro, o membro contralateral a $\mathrm{MN}$ permanecia em flexão para estabilizar a lombar. 
Logo em seguida a aplicação da mobilização neural, realizou-se novamente a avaliação goniométrica e da EVA, na mesma sequência da avaliação pré-mobilização, com a finalidade de distinguir diferenças, em graus e redução da dor da pré e pós-MN na dor lombar. Os dados da EVA e os graus de ADM da flexão de tronco pré e pós-mobilização neural foram anexados na ficha de cada participante, sempre iniciando a MN pelo membro inferior esquerdo para assim fixar um padrão no estudo.

A tabulação dos dados foi feita em Microsoft Excel, atribuindo códigos numéricos específicos às variáveis categóricas. Os pacotes estatísticos utilizados para as análises de dados foram o SPSS 20.0 e o BioEstat 5.0 (versão em português). Foram feitas análises descritivas das variáveis contínuas, como frequências absolutas e relativas, média, desvio-padrão, mínimo e máximo. O teste de normalidade realizado foi o Kolmogorov-Smirnov. Seguiu-se as análises paramétricas de comparação de médias teste t de Student pareado e independente (em situações diferentes), além da análise de variância (ANOVA), com diferenciação das médias utilizando o teste Tukey. Para todos os testes estatísticos, utilizou-se o nível de significância de 5\%.

\section{Resultados}

A amostra foi constituída por 08 indivíduos voluntários, 2 (25\%) do sexo masculino e 6 (75\%) do sexo feminino, com idade média de 55,25 \pm 15,62 anos, estatura média de 1,62 \pm 0,10 metros e peso médio de 27,32 $\pm 5,83 \mathrm{~kg}$, com localização d hérnia lombar 6 (75\%) L4/L5 e 2 (25\%) L5/S1, conforme os dados da tabela 1.

Tabela 1. Perfil sócio demográfico dos indivíduos com dor lombar e quadro de hérnia de disco. Vitória da Conquista - BA, 2017.

\begin{tabular}{lllll}
\hline Variável & Média & Desvio-padrão & Mínimo & Máximo \\
\hline Idade & 55,25 & 15,62 & 30,00 & 69,00 \\
Peso & 71,81 & 17,08 & 50,00 & 100,00 \\
Altura & 1,62 & 0,10 & 1,50 & 1,80 \\
IMC & 27,32 & 5,83 & 20,03 & 35,86
\end{tabular}

Fonte: Dados da pesquisa 
Os dados referentes à dor lombar identificaram 4 (50\%) indivíduos com dor moderada e 4 (50\%) indivíduos com dor intensa através da Escala Visual Analógica e o questionário STarT Back Screening Tool (SBST) auxiliou na triagem desses pacientes em relação ao risco de mau prognóstico no tratamento primário, considerando fatores físicos e psicossociais identificando $5(62,50 \%)$ indivíduos com baixo risco e $3(37,50 \%)$ indivíduos com médio risco.

Os descritores do Questionario McGill que melhor caracterizavam a dor dos participantes foram: vibração (30\%), tremor (10\%), pulsante (20\%), latejante (40\%), formigamento $(50 \%)$, coceira $(16,67 \%)$, ardor $(33,33 \%)$, chata $(50 \%)$, que incomoda $(40 \%)$, desgastante $(10 \%)$, espalha (20\%), que irradia (60\%), que penetra (10\%) e que atravessa (10\%). A média do numero de palavras escolhidas pelos participantes foi de 19,00 palavras e desvio-padrao 5,37, sendo o mínimo 10 e o máximo 29.

A avaliação da amplitude de movimento de flexão de tronco mostrou normalidades dos dados, de acordo com o teste $t$ pareado. A média da flexão de tronco pré-MN do nervo isquiático foi de $5,80 \pm 40,80^{\circ}$ e pós-MN de $5,25 \pm 39,34^{\circ}$, demonstrando um aumento flexão de tronco após a aplicação da MN com uma diferença estatisticamente significante $(\mathrm{p}<0,01)$ pelo teste t de Student para dados pareados, como representa no gráfico 1.

Gráfico 1. Comparando a mobilidade funcional antes e após o tratamento. Vitória da Conquista - BA, 2017.

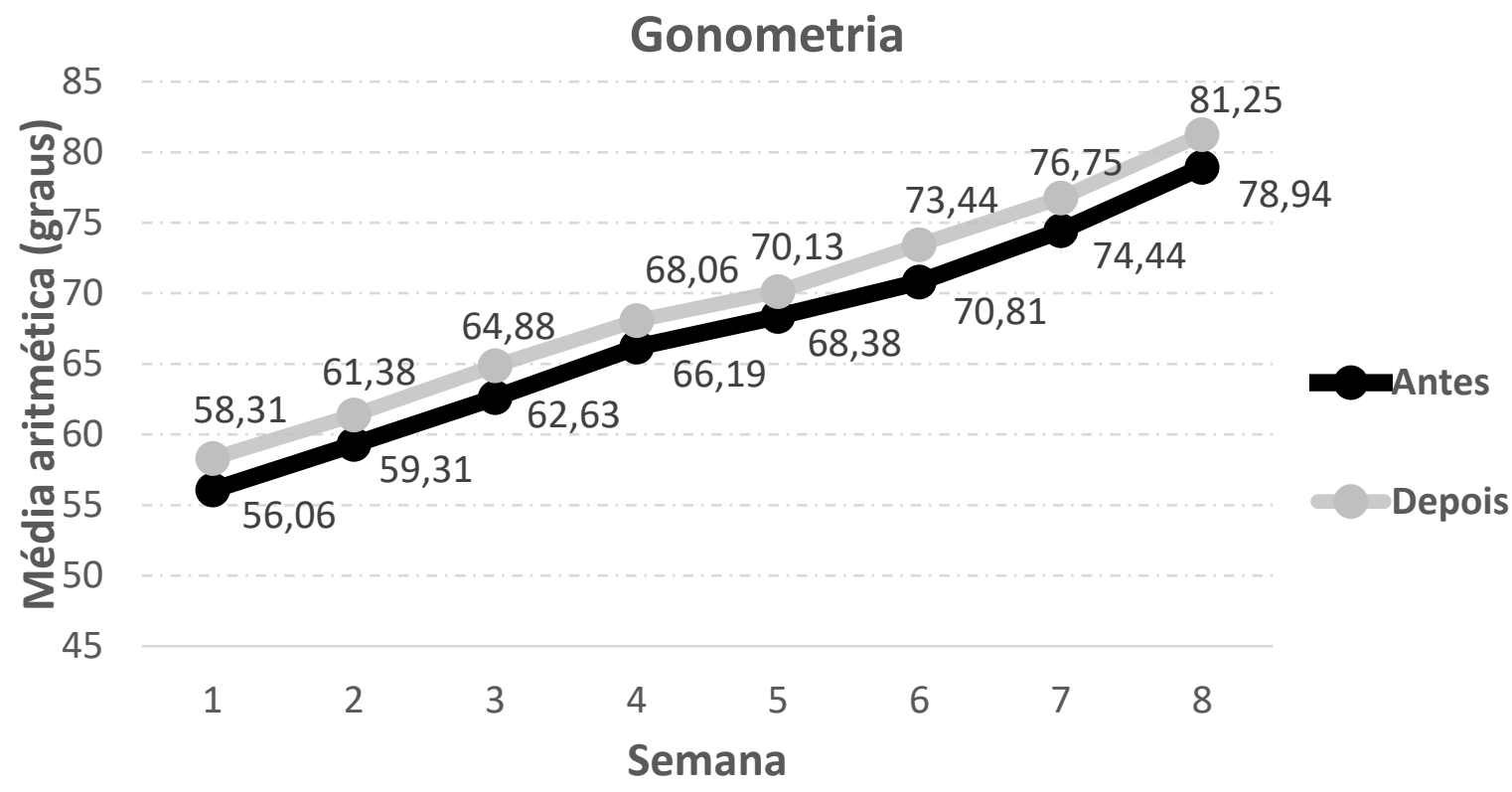

Fonte: Dados da pesquisa. 
Também achou-se diminuição significativa na variável dor, onde a mesma foi avaliada pela EVA da segunda sessão em relação à primeira, diminuiu em média da escala de dor 18,45\%. Para a última sessão, a redução da dor foi de 79,61\% (Gráfico 2).

Gráfico 2. Verificou a intensidade da dor lombar pré e pós MN. Vitória da Conquista - BA, 2017.

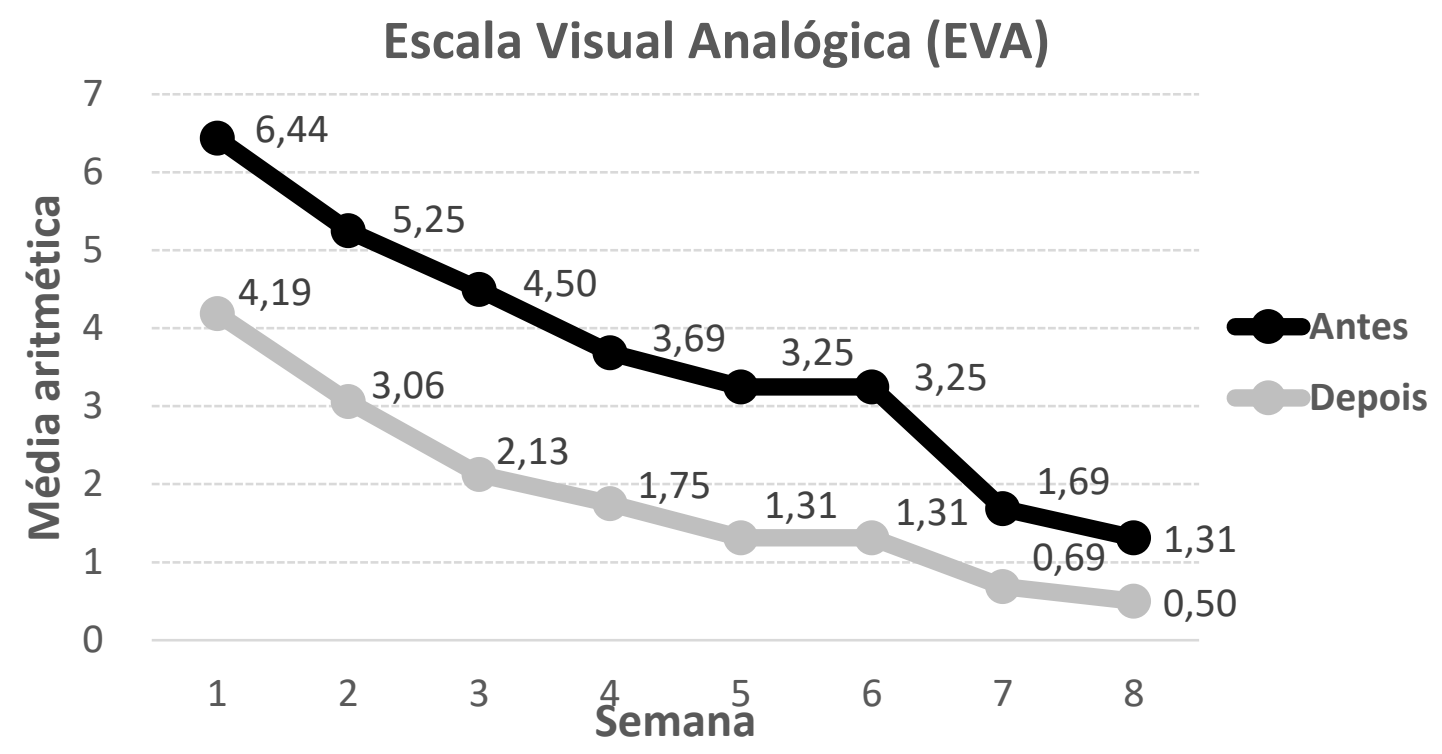

.Fonte: Dados da pesquisa.

\section{Discussão}

Nos últimos anos, alguns estudos buscam uma avaliação em relação aos efeitos que a mobilização neural pode causar em varias disfunções do corpo. No presente estudo analisou os efeitos dessa técnica em lombalgias nos indivíduos com hérnia discal lombar. O tratamento de mobilização neural do sistema nervoso vem sendo explorado como uma técnica de avaliação e tratamento dentre diversas patologias que supostamente acometem o sistema nervoso e as estruturas que são inervadas por ele. Pacientes com dor lombar tratados com mobilização neural conseguiram uma redução da sintomatologia dolorosa, melhora da qualidade de vida e retorno às atividades de vida diária ( LIMA et al., 2012). 
O Sistema Nervoso é formado por propriedades elásticas, tendo na sua capacidade o poder de se encurtar ou se alongar. Ao realizar o movimento de flexão da coluna vertebral, temos o aumento do comprimento de todas as estruturas neurais espinhais. Com isso ocorre um aumento no canal medular que chega ate $9 \mathrm{~cm}$ de comprimento durante o movimento de flexão da coluna inteira. Sedo assim afirma-se que a tensão do tecido neural poderá ser aumentada, com uma flexão de pescoço, ou com um movimento de dorsi-flexão do tornozelo (JUNIOR; MONNERAT, 2012).

Diante disso essa técnica, parte-se do princípio que comprometimentos da fisiologia e da mecânica do sistema nervoso (movimento, elasticidade, condução, fluxo axoplasmático) podem gerar alterações do sistema nervoso, ou nas estruturas musculoesqueléticas que se inerva por ele, e que o restabelecimento de sua biomecânica e fisiologia adequada, por meio de oscilações ou tensão, promove a recuperação da extensibilidade e da funcionalidade normal desse sistema e das estruturas comprometidas (BARBOSA; LEAL, 2015).

Uma das variáveis que expôs diferença significativa, quando avaliada antes e após a aplicação da mobilização neural, foi a amplitude de movimento de coluna lombar. O que corrobora com o estudo de (JUNIOR; SCHONS, 2015), que avaliaram 11 pacientes, 6 do sexo feminino e 5 do sexo masculino antes e após a mobilização neural para ganhar amplitude de movimento. Em relação à ADM, na avaliação inicial os indivíduos apresentaram uma média de $41,81^{\circ}( \pm 7,54)$. Na avaliação final essa média foi de $55^{\circ}( \pm 9,58)$, expressando assim um aumento significativo da ADM ( $\mathrm{p}=0,0007)$.

Em outro estudo realizado pode verificar os efeitos imediatos da MN do nervo mediano sobre o ganho de amplitude de movimento de extensão do cotovelo em 60 indivíduos neurologicamente assintomáticos e observou-se que houve ganho médio de $11,04^{\circ}$ no membro superior direito, variando de uma média de $31,57^{\circ}$, pré-mobilização, para $20,53^{\circ}$ pósmobilização, e $12,11^{\circ}$ no membro superior esquerdo, variando de $28,68^{\circ}$ para $16,57^{\circ}$ pré- e pósmobilização, respectivamente. Esses resultados mostraram a eficácia da técnica de MN no aumento da amplitude de movimento em indivíduos com tensão neural adversa do presente estudo (VASCONCELOS; LINS; DANTAS, 2011).

De acordo com Barbosa e Leal (2015), ao analisar 50 indivíduos, divididos em dois grupos. No Grupo 1, realizou-se avaliação da amplitude do membro direito, em seguida, executaram-se aplicação da técnica de MN e reavaliação da ADM também no referido membro. No Grupo 2, efetuaram-se avaliação do membro direito, aplicação da técnica de MN no esquerdo e reavaliação da ADM no direito. Foi possível notar uma diferença estatisticamente 
significativa entre o antes e o depois da realização da técnica de mobilização neural, apresentando $\mathrm{p}=0,001$. Verificou-se, assim, a capacidade do método no ganho da amplitude de movimento.

De acordo com o presente estudo a variável que houve maior significância foi em relação à dor. Segundo Freitas et al., (2015) em seu estudo foram selecionados oito pacientes com lombociatalgia divididos aleatoriamente em dois grupos: G1 realizou fortalecimento de glúteos e estabilização e G2 realizou a conduta do G1 adicionado a mobilização neural do nervo ciático. Os participantes tiveram como método de avaliação para dor a EVA.

Ao avaliar o quadro álgico da coluna lombar através da EVA, o G1 apresentou media de dor pré-tratamento de 5,5 $\pm 1,9$, e o G2 apresentou media de 4,5 $\pm 0,5$. Após 8 semanas de tratamento, a media de dor no G1 diminuiu para 1,75 $\pm 2,0$ sendo diferente estatisticamente da media pré-tratamento $(\mathrm{p}<0,03)$.

Já em um estudo conduzido por Junior; Schons, (2015) com 11 pacientes reacionado aos efeitos da Mobilização Neural com os pacientes com lombociatalgia, não houve influência em relação à sintomatologia através da EVA, no pré-tratamento os participantes apresentaram uma média de 3,81( $\pm 2,19)$, e na avaliação final 3,36 $( \pm 2,41)$, notando-se leve melhora após a aplicação da técnica, mas sem diferença significativa $(\mathrm{p}=0,59)$. Porém os autores resaltam que precisa-se levar em conta que 2 pacientes finalizaram o tratamento com graduação da EVA em 0 .

De acordo com o estudo de Monnerat et al,. (2012), sobre o efeito da MN na melhora da lombalgia e limitação funcional da hérnia de disco lombar subaguda ele demonstra que a técnica de Mobilização Neural e eficaz no tratamento da hérnia de disco lombar em fase subaguda por apresentar boa resposta terapêutica na redução das sintomatologias e limitação funcional em curto período de tempo, quando comparada ao tratamento fisioterapeutico convencional.

Para esta mesma disfunção, 12 sessões utilizando a técnica de Mobilização Neural foram suficientes para redução da sintomatologia dolorosa e melhora da capacidade funcional (MONNERAT; JÚNIOR, 2012).

Como limitação deste estudo cita-se o fato do tamanho da amostra não ser tão expressivo, portanto não se pode generalizar para a população. Existe a precisão de serem realizados mais estudos sobre o tema para que haja melhor aprimoramento e validação da mobilização neural. 


\section{Conclusão}

A partir dos resultados obtidos no presente estudo conclui-se que os indivíduos participantes da pesquisa tiveram um ganho da mobilidade funcional e redução do quadro álgico através da Mobilização Neural, obtendo-se relação estatística significativa na dor lombar através da Escala Visual Analógica e goniometria.

A Mobilização Neural apresentou um alto índice de melhora nos pacientes tratados, daí uma importância pra os indivíduos por ser um método conservador ela vem se destacando na pratica clinica para varias alterações músculo esqueléticas. Como foi discutindo por vários estudos ela proporciona uma redução na dor lombar e aumento da mobilidade dos indivíduos favorecendo uma melhor qualidade de vida.

\section{Referências}

BIAUCHL, A. B. et. al. Estudo comparativo entre os métodos Pilates no solo e Water Pilates na qualidade de vida e dor de pacientes com lombalgia. Revista do Departamento de Educação Física e Saúde e do Mestrado em Promoção da Saúde da Universidade de Santa Cruz do Sul / Unisc, v. 17, n. 4, Outubro/Dezembro 2016.

FREITAS, C. A. et. al. Importância clínica da Mobilização Neural em pacientes com lombociatalgia. Fisioterapia Brasil, v. 16, n. 1, 2015.

KOBILL, A. F. M. Influência da Estabilização Segmentar core na dor e funcionalidade da coluna lombar. Fisioterapia Brasil, v. 18, n. 2, p. 148-153, 2017.

MENDES, P. M. Aplicação da escala de McGill para avaliação da dor em pacientes oncológicos. Revista de Enfermagem UFPE on line, v. 10, n. 11, p. 4051-7, nov 2016.

MENDONÇA, E. M. T.; ANDRADE, T.M. Método Mckenzie como protocolo de tratamento em hérnia de disco lombar. Revista Interdisciplinar, v. 9, n. 3, p. 130-137, jul. ago. set. 2016.

PILZ, B. The Brazilian version of STarT Back Screening Tool - translation, cross-cultural adaptation and reliability. Brazilian Journal of Physical Therapy, v. 18, n. 5, p. 453-461, 2014.

STORCH, J. et. al. O método Pilates associado a orientações de estilo de vida em pacientes com lombalgia crônica. LifeStyle Journal, v. 2, n. 2, p. 53-66, 2015. 
MONNERAT, E. et. al. Efeito da Mobilização Neural na melhora da dor e incapacidade funcional da hérnia de disco lombar subaguda. Fisioterapia Brasil, v. 13, n.1, janeiro/fevereiro de 2012.

BARBOSA, A. P. B.; LEAL, S. S. Análise da eficácia da mobilização neural do nervo isquiático sobre ganho de ADM. Revista ConScientiae Saúde, v. 14, n. 3, p. 463-469, 2015

JUNIOR, P. C. N.; MONNETAT, E. Comparação dos tratamentos conservador, cirúrgico e através da mobilização neural no tratamento da hérnia de disco lombar. Fisioterapia Brasil, v. 13, n. 2, 2012.

KODAMA, F. Y. et, al. Estudo do ângulo Q via fotometria e goniometria em atletas profissionais de futsal. Revista Saúde UniToledo, Araçatuba, SP, v. 01, n. 01, p. 64-75, mar./ago. 2017

LIMA, M. O. et, al. A eficiência da mobilização neural na reabilitação da lombalgia: uma revisão de literatura. Revista Brasileira de Ciências da Saúde, v. 10, n. 31, jan/mar 2012.

JUNIOR, A. A. P.; SCHONS, D. G. Os efeitos da Mobilização Neural em Pacientes com lombociatalgia. Revista Fisioterapia e Saúde Funcional. Fortaleza, v. 4, n. 2, p. 14-20, JulDez. 2015.

VASCONCELOS, D. A.; LINS, L. C. R. F.; DANTAS, E. H. M. Avaliação da mobilização neural sobre o ganho de amplitude de movimento. Fisioterapia e Movimento, v. 24, n. 4, p. 665-672, out./dez. 2011.

FERREIRA, J. N. S.; OLIVEIRA, A. S. Estabilização segmentar vertebral em pacientes com lombalgia crônica: revisão da literatura. Corpussci, Rio de Janeiro, v. 11, n. 2, p . 24 - 32 , jul /dez . 2015.

SIQUEIRA, G. DE et al. A Eficácia Da Estabilização Segmentar Vertebral No Aumento Do Trofismo Dos Multífidos Em Portadores De Hérnia Discal Lombar. Revista Brasileira de Ciência e Moviento, v. 22, n. 1, p. 81-89, 2014.

\section{Como citar este artigo (Formato ABNT):}

FERREIRA, Adirlane M.; SANTANA JÚNIOR, Virgilio. Análise do efeito da Mobilização Neural na dor lombar em pacientes com hérnia de disco. Id on Line Revista Multidisciplinar e de Psicologia, 2017, vol.11, n.38, p.824-834. ISSN: 1981-1179.

Recebido: 08.11.2017

Aceito: 10.11 .2017 